\title{
Early insulin resistance is associated with alterations in gut permeability and microbial activity in rats fed a moderately westernized diet
}

\section{Abstract}

In Western societies, unbalanced diets may lead to intestinal allostatic stress, with microbial dysbiosis and alteration of the gut barrier function. These local modifications may induce a low-grade inflammation via endotoxemic process, which contribute to systemic alterations clustering into the metabolic syndrome (MetS) and increasing the risk of cardiovascular diseases and type 2 diabetes. We investigated the sequence of events initiating the MetS in rats challenged with a diet that differed moderately from the dietary guidelines, regarding the proportions and nature of energy, carbohydrates, lipids and fibre.

Male Wistar rats (265 g upon arrival) were with either a standard diet (Con, $\mathrm{n}=10,3730 \mathrm{kcalkg}, 4.0 \%$ lipids, $6.3 \%$ sucrose, 58.0\% starch, $5.0 \%$ fibre) or a westernized induction diet (Ind, $\mathrm{n}=10$, moderately enriched in energy (4120 kcal $/ \mathrm{kg})$, lipid (24.0\%), and sugars $(15 \%$, half sucrose-half fructose), and low in starch (25.5\%) and fibre (1.25\%). An oral glucose tolerance test (OGTT) was performed after 3 weeks. Rats were euthanized after a further week for body composition and collection of caecum content and tissue samples. LPS-binding protein (LPS-BP) was assayed in plasma; ileum and colon samples were used for measurement of wall barrier function (using Ussing chambers) and microbial activity was assessed in caecum (enzymes and metabolites).

Body weight did not differ between Con and Ind groups at any time, as well as the absolute masses and proportions of visceral adipose tissue and liver. Fasting triglyceride, glucose, insulin and LPS-BP concentrations, HOMA index and glucose AUC during the OGTT were similar in both groups. When compared to the Con group, Ind rats showed significantly higher risk markers, i.e.: (1) Insulin AUC (x2); (2) Hepatic triglyceride content (x2.8); (3) Wall permeability at both the para- and transcellular levels (x2.6 and 4.3, respectively) in the ileum (but not in the colon). In the caecum, $\mathrm{H}_{2} \mathrm{~S}$ absolute and relative amounts were twice higher in the Ind group, in relation with 2-3-fold increases in specific and total activities of microbial cysteine desulfhydrase and proteases.

Multiple dietary changes mimicking a moderately westernized diet induced an increased ileal permeability and changes in the caecal microbial activity, without evidence of endotoxemia. These perturbations were associated with detrimental rupture of homeostasis at both the splanchnic (hepatic steatosis) and systemic (insulin resistance) levels. In this realistic model, these alterations preceded the occurrence of clinical markers of the MetS (obesity and elevated plasma concentrations of glucose and triglyceride).

Conflict of Interest

There is no conflict of interest 